\title{
The Impact of Population-Based Disease Management Services on Health Care Utilisation and Costs: Results of the CAPICHe Trial
}

\author{
Paul A. Scuffham, $P h D^{7}$, Joshua M. Byrnes, $P h D^{7}$, Christine Pollicino, M Med Stat ${ }^{2}$, David Cross, BSc ${ }^{3}$, \\ Stan Goldstein, MBBS, MHA ${ }^{2}$, and Shu-Kay $\mathrm{Ng} \mathrm{PhD}^{7}$
}

${ }^{1}$ Centre for Applied Health Economics, Menzies Health Institute Queensland, Griffith University, Brisbane, Australia; ${ }^{2}$ Bupa Australia, Sydney, Australia; ${ }^{3}$ Bupa Health Dialog, Melbourne, Victoria, Australia.

\begin{abstract}
BACKGROUND: Disease management programmes may improve quality of care, improve health outcomes and potentially reduce total healthcare costs. To date, only one very large population-based study has been undertaken and indicated reductions in hospital admissions $>10 \%$.

OBJECTIVE: We sought to confirm the effectiveness of population-based disease management programmes. The objective of this study was to evaluate the relative impact on healthcare utilisation and cost of participants the Costs to Australian Private Insurance - Coaching Health (CAPICHe) trial.
\end{abstract}

DESIGN: Parallel-group randomised controlled trial, intention-to-treat analysis

SETTING: Australian population

PARTICIPANTS: Forty-four thousand four hundred eighteen individuals (18-90 years of age) with private health insurance and diagnosis of heart failure, chronic obstructive pulmonary disease (COPD), coronary artery disease (CAD), diabetes, or low back pain, with predicted high cost claims for the following 12 months.

INTERVENTION: Health coaching for disease management from Bupa Health Dialog, vs Usual Care.

MAIN OUTCOME MEASURES: Total cost of claims per member to the private health insurer 1 year postrandomisation for hospital admissions, including sameday, medical and prostheses hospital claims, excluding any maternity costs. Analysis was based on the intentto-treat population.

RESULTS: Estimated total cost 1 year post-randomisation was not significantly different (means: intervention group A\$4934; 95\% CI A\$4823-A\$5045 vs control group A\$4868; 95\% CI A\$4680-A\$5058; $p=0.524)$. However, the intervention group had significantly lower same-day admission costs (A\$468; 95\% CI A\$454-A\$482 vs A\$508; 95\% CI A\$484-A\$533; $p=0.002$ ) and fewer same-day

To date, there have been no conference presentations of this study; all presentations have been to Bupa Australia and Bupa Health Foundation only.

Electronic supplementary material The online version of this article (https://doi.org/10.1007/s11606-018-4682-5) contains supplementary material, which is available to authorized users.

Received December 26, 2017

Revised July 6, 2018

Accepted September 12, 2018

Published online September 27, 2018 admissions per 1000 person-years (intervention group, 530; 95\% CI 508-552 vs control group, 614; 95\% CI 571$657 ; p=0.002$ ). Subgroup analyses indicated that the intervention group had significantly fewer admissions for patients with COPD and fewer same-day admissions for patients with diabetes.

CONCLUSIONS: Chronic disease health coaching was not effective to reduce the total cost after 12 months of followup for higher risk individuals with a chronic condition. Statistically significant changes were found with fewer same-day admissions; however, these did not translate into cost savings from a private health insurance perspective.

KEY WORDS: disease management; costs; private healthcare; insurance.

J Gen Intern Med 34(1):41-8

DOI: $10.1007 / \mathrm{s} 11606-018-4682-5$

(C) Society of General Internal Medicine 2018

\section{INTRODUCTION}

Disease management is a broad term that describes a range of activities designed to mitigate the progression and impacts of health conditions and encourage adherence to treatment regimens and self-care strategies. ${ }^{1}$ Disease management programmes have been promoted as a way to potentially improve quality of care and health outcomes and reduce healthcare expenditure. $^{2-4}$

To date, the effects of disease management programmes on healthcare expenditure have been mixed. Beneficial claims of disease management programmes have been criticised on the grounds that they have generally involved highly selected participants in closed systems of care, or suffered from a number of biases, such as bias in the recruitment or enrolment of participants. ${ }^{5}$ To address the apparent lack of large-scale, methodologically rigorous investigations into the effects of disease management on financial outcomes, a randomised trial of telephone care management within the USA was conducted for a trial population of 174,120 participants, of which 86,877 randomised to the intervention group and 9035 received coaching. ${ }^{6}$ That trial indicated that their disease management programme reduced health costs and utilisation of healthcare resources. 
In view of the limited and conflicting international evidence and the lack of evidence in Australia, the Costs to Australian Private Insurance - Coaching Health (CAPICHe) trial was undertaken. Over 3.75 million patients were initially assessed for eligibility for the CAPICHe trial. A total of 44,418 individuals were ultimately randomised to the trial and 35,535 were in the intervention group, of which 15,375 people received health coaching. Based on the number of people receiving coaching, this is the largest controlled trial of its type ever conducted. The objective of the CAPICHe trial was to evaluate the relative impact on healthcare utilisation and costs of participants in a disease management programme provided through telephone-based health coaching support compared to usual care. This article reports the outcomes from the CAPICHe trial for the intention-to-treat population.

\section{METHODS}

\section{Trial Design and Participants}

The trial protocol has previously been published. ${ }^{7}$ The trial was approved by Griffith University Human Research Ethics Committee (Ref: MED/12/11/HREC) and registered with the Australian New Zealand Clinical Trials Registry, number ACTRN12611000580976. Figure 1 summarises recruitment, randomisation and follow-up processes of the trial. CAPICHe was a parallel-group randomised controlled trial. The trial enrolled participants sourced from the approximately four million Bupa Australia health fund members located across Australia. Bupa provides private health and hospital insurance to all persons that seek it in Australia. To reduce the risk of contamination of results from other disease management programmes available to the health fund's members, a series of inclusion and exclusion criteria were applied prior to randomisation (Fig. 1).

The trial was designed to target the population that has a high likelihood of further hospitalisation and health costs in the short term. Pre-specified inclusion criteria consisted of (a) 18-90 years of age; (b) held hospital cover with Bupa Australia; (c) had a valid Australian mailing address; (d) had claims evidence of diagnosis of heart failure, chronic obstructive pulmonary disease (COPD), coronary artery disease (CAD), diabetes, or low back pain (or more than one of these); (e) the highest risk member in the household; and (f) had a predicted cost for any cause (ascertained by a Bupa Health Dialog proprietary claims-based risk model based on previous medical and utilisation history) for the following 12 months of \$3163 or more, in 2012 Australian dollars (i.e. members who would be likely to use health care services in the future). This cut-off was decided to achieve a larger sample size of randomised at risk participants. Where a patient was identified with more than one condition, he/she was allocated to the highest order condition based on a clinical hierarchy of heart failure, COPD, CAD, diabetes, or low back pain, which is consistent with the ICD-10 classification system. The number of other

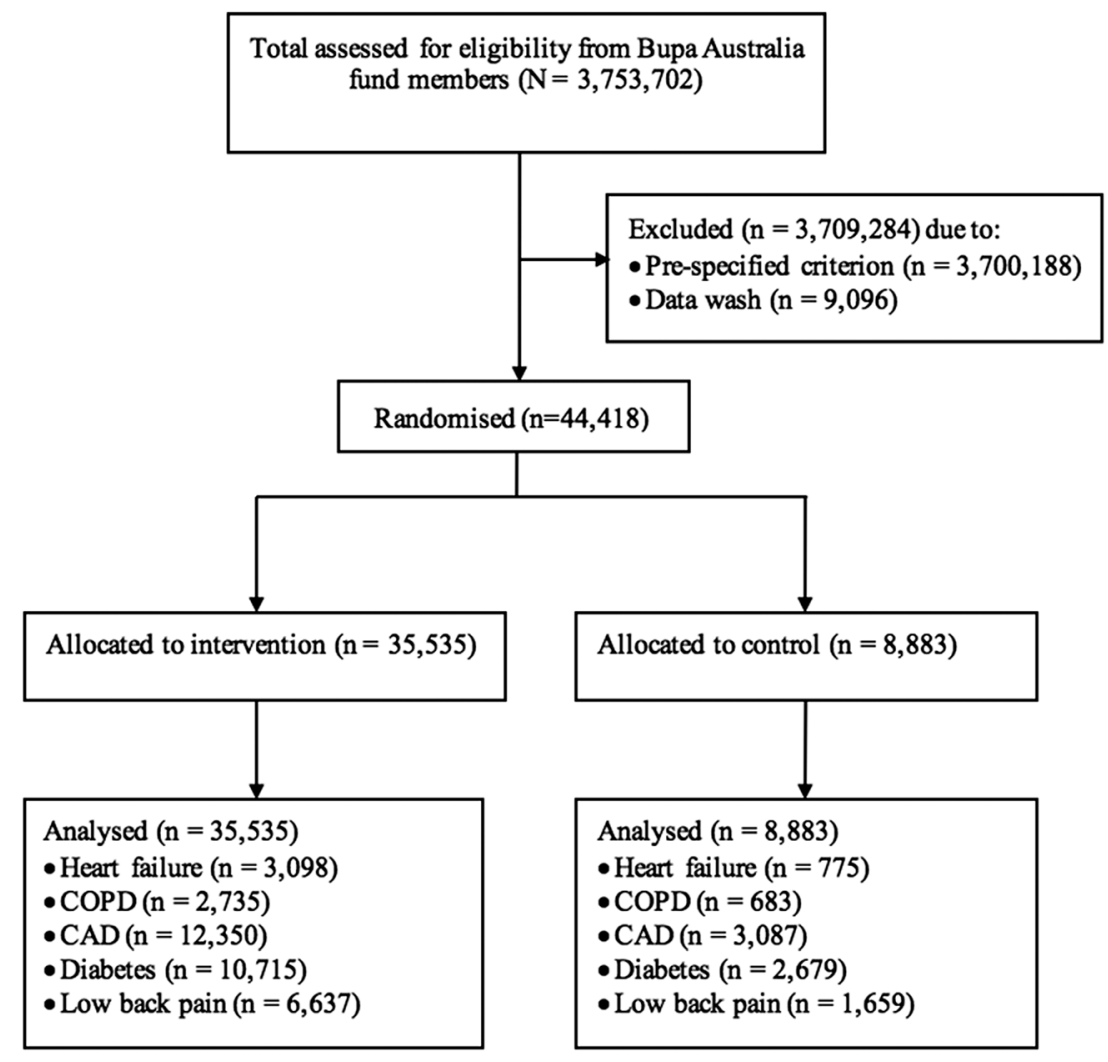

Figure 1. CONSORT flowchart: recruitment, randomisation, and follow-up of participants. COPD= chronic obstructive pulmonary disease. CAD, coronary artery disease; ITT, intention-to-treat. 
diagnosed conditions counted in the hierarchy was used as a covariate in the analysis.

Exclusion criteria were (a) shared a household with a previously assigned trial participant; (b) were targeted for and/or involved in potentially similar services prior to trial initiation; and (c) had claims evidence in the past 12 months prior to trial initiation of condition including end-stage renal disease, human immunodeficiency virus infection and acquired immune deficiency syndrome (HIV/AIDS), cancer treatment, or an organ transplant.

The participants in the intervention group were eligible to receive disease management services from Bupa Health Dialog. In the control group, the participants received a letter outlining the service provided by Bupa Health Dialog. Generally, these participants received usual care but were given the opportunity to "opt-in" to receive health coaching. If a participant assigned to the usual care group actively sought engagement with a health coach, they received the same service as that provided to a participant assigned to the intervention group (i.e. health coaching). Health coaches had the same information for all those receiving coaching and all had the same educational resources made available to them. Detailed procedures of the intervention are provided in Section A of the online-only text. Briefly, the intervention included programme awareness notifications, outbound Health Coach Outreach, and follow-up calls (a minimum of one call in the first 2 months with no maximum number of calls being set), access to health coaches via telephone as required and tailored outreach and educational materials. The health coaching package was based on that described previously by Wennberg et al. after modification for use in Australia. ${ }^{6}$

\section{Randomisation and Masking}

Each month, participants who met the inclusion criteria were randomly selected from Bupa Australia claims database. The samples were then randomised into the intervention or control groups in a 4:1 ratio stratified by chronic condition, where a larger sampling ratio for intervention is to maximise the coverage for those to receive the intervention. ${ }^{7}$ The effectiveness of randomisation for each monthly batch of data was checked. Randomisation was undertaken by the Griffith University Clinical Trials Unit (www.griffith.edu.au/health/centre-health-practice-innovation/research/griffith-clinical-trials-unit-ctu), and independent of Bupa Australia. Other than participants in the usual care arm who opted-in to the intervention and received health coaching, the health coaches were blind to whether participants were in the CAPICHe intervention group or were receiving health coaching as part of the usual business for Bupa.

\section{Outcomes}

The primary trial outcome was the total cost per member as reported to the private health insurer 1 year post-randomisation for all impactable hospital admissions, excluding any maternity costs. Non-impactable admissions were excluded (eTable 1).
The cost (i.e. the total benefit paid by the health insurer) was calculated as the sum of hospital, medical (excluding the $75 \%$ Medicare Benefits Schedule component paid by the Australian Government) and prostheses claims. Member contributions (in the form of an excess or co-payments), ancillary benefits such as dental and optical and the cost of providing the intervention were not included in the analysis.

Secondary outcomes were costs in the intervention year per member by stay type (overnight or same day) and rates of inpatient bed days in the intervention year, excluding any maternity costs and utilisation. An overnight admission is one where the patient is admitted and separated (i.e. discharged, transferred to another institution or died) on a different day (i.e. stays at least one night). For the purpose of this analysis, same-day admissions include both non-admitted outpatient services such as emergency department visits (where patients do not stay overnight and are not admitted to hospital) and same-day admissions where patients are formally admitted to hospital. The latter is analogous to an outpatient visit in the USA. The primary reasons for same-day admissions with a formal admission process in Australia are care involving dialysis, other medical care (primarily chemotherapy for cancer) and cataract procedures, ${ }^{8}$ plus other investigative procedures that may require a general anaesthetic (e.g. endoscopies). These admissions are predominantly elective (around 70\%).

Claims data were extracted from Bupa Australia data systems. Each trial participant was followed continuously through claims data collection until paid claims had accrued for a full 12-month period after randomisation. An additional 4 months was allowed for the processing of claims to ensure the overwhelming majority of insurance claims data had been collected and processed.

\section{Statistical Analysis}

The sample size calculation was revised since the publication of the trial protocol ${ }^{7}$ due to achieving a better engagement rate than presumed in a pilot study. The revised calculations were based on two assumptions: (a) at least $38 \%$ of those randomised to the intervention group were engaged with a health coach (compared to initial assumption of 30\%) and (b) those engaged had a $12.5 \%$ reduction in healthcare claims over the 12 months of follow-up compared with the control group (consistent with initial assumption). This gave an overall expected effect size of $4.75 \%$ for the intervention group (compared to an initially expected effect size of $3.75 \%$ ). To detect this difference in total costs at the $5 \%$ level of significance with $80 \%$ power and a coefficient of variation of 2.42 (based on historical claims), an overall sample size of 39,880 participants was determined. Allowing for $8 \%$ of health fund members who lose eligibility through cessation of health insurance or death (estimated based on experience with the general insured population), a sample of at least 43,350 participants were needed for the trial (compared to an initial estimate of 
64,835). The effect sizes that can be detected for pre-planned subgroup analyses were given in Section A of the online-only text.

Statistical analysis was performed in Stata (Stata IC 13.1; StataCorp, College Station, TX), with an intention-to-treat approach to study intervention effectiveness based on comparisons between assigned trial arms regardless of whether the participants complied with the assigned protocol (discussion on alternative approaches including per-protocol analysis is available). ${ }^{9,}{ }^{10}$ Because there was an excess number of observations with zero costs or zero admissions, zero-inflated regression models, ${ }^{11}, 12$ with either logit or probit for characterising excess zeros, were adopted to compare the risk of excess zero costs and the mean costs (primary and secondary outcomes) between the two groups. The best model was assessed based on goodness-of-fit and Bayesian Information Criterion (BIC). Sensitivity analyses to compare the results between zero-inflated models and two-part models ${ }^{13}$ were performed. Covariates including historical costs (within 12 months prior to randomisation) by service category, historical admission counts, age, gender, state of residence and potential batch effects, as well as the diagnosed chronic condition, the count of chronic conditions, the number of follow-up days from randomisation and the proportion of admissions due to surgical treatment within the follow-up period, were included in the regression models. Interaction terms between covariates and the intervention group were considered in all data analyses. Data from participants who ceased private health insurance before 1 year post-randomisation were adjusted using the observed days of follow-up as exposure risk. These procedures thus ensure that potential source of bias due to contamination in the two trial arms was accounted for in the regression analyses. Alternatively, a contamination-adjusted intentionto-treat approach ${ }^{10}$ may be used to assign treatment as an instrumental variable (IV) for provision of complementary information regarding the effectiveness of the disease management programmes. With a forward stepwise procedure, covariates were added to or removed from the models based on the 0.05 levels of significance in this study. We also performed pre-planned subgroup analyses, defined by the five diagnosed chronic conditions and two age groups (age $<65$ years and age over 65 years). All costs are reported in Australian dollars (AUD) 2012 dollar values (in 2012, A $\$ 1 \approx £ 0.65 \approx$ US\$1.04).

\section{RESULTS}

A total of 3,753,702 Bupa Australia fund members were assessed for eligibility. Of these, a total of 44,418 participants were enrolled in the trial, of whom 35,535 participants $(80 \%)$ were allocated to the CAPICHe intervention group and 8883 participants $(20 \%)$ were allocated to the usual care control group. Participant characteristics did not differ between the two groups, except those randomised to the intervention group had slightly fewer admissions (any type) within 12 months prior to randomisation (Table 1). There was no difference in mortality rates between the two groups $(p=0.941)$. The trial arms had a balanced and broad geographical distribution of participants across Australia. There was no difference in the number of overnight-only admissions within 12 months from randomisation between the two groups. However, participants in the intervention group had fewer same-day only admissions (especially admissions due to medical treatment) within 12 months from randomisation (Table 1).

Of the participants allocated to the intervention group, $43.3 \%(15,375 / 35,535)$ were engaged and had a median of 2 coaching sessions (inter-quartile range 2-4; mean 3.2); the median time to first contact was 87 days from randomisation. Of the participants allocated to the usual care group, $1.7 \%$ (153/ 8883) chose to receive health coaching (the median time to first contact was 49 days from randomisation; a median of three contacts). This proportion of opt-in participants was minor.

Estimated total cost 1 year post-randomisation was slightly higher for the intervention group (mean $\$ 4934 ; 95 \%$ CI $\$ 4823$ to $\$ 5045$ ) compared to the control group ( $\$ 4868 ; 95 \% \mathrm{CI}$ $\$ 4680$ to $\$ 5058$ ), which was not significant with $p=0.524$ (see eTable 2 for analysis result). The difference in total cost in the intervention group relative to the control group was $\$ 66$ (95\% CI \$136 to \$267) (see Table 2). Results were found to be consistent across a number of different statistical models. For the subgroup analyses (Fig. 2 and eTable 3), there were no significant differences in total cost between the intervention group and the control group for all five chronic conditions (i.e. heart failure, COPD, coronary artery disease, diabetes, or low back pain). However, the intervention group had significantly lower cost due to same-day admission (difference of $\$ 40 ; 95 \%$ CI $\$ 16$ to $\$ 64 ; p=0.001)$.

The rate of same-day admissions was also significantly lower for the intervention group (difference of 85 per 1000 person-years; 95\% CI 40 to 129 per 1000 person-years; $p<0.001)$. Patients with diabetes in the intervention group had a lower cost for same-day admissions (intervention group, $\$ 464$ versus control group, $\$ 550 ; p<0.001)$ as a result of significantly fewer same-day admissions (rate per 1000 person-years, 612 versus $758 ; p=0.007$ ). The intervention group also had fewer admissions (all types) for patients with COPD (1531 versus 1882 per 1000 person-years; $p=0.010$ ). Subgroup analyses by age group showed (marginal) significantly lower total health care costs ( $\$ 3721$ versus $\$ 4105 ; p=0.063$ ), lower same-day admission costs $(\$ 480$ versus $\$ 602$; $p<0.001)$ and fewer same-day admissions (661 versus 1029 per 1000 person-years; $p<0.001)$ for participants aged under 65 years (eTable 4). No clinical basis as to why health coaching may lead to a reduction in same-day admissions could be established through the analysis of the data.

Additional results of sensitivity analyses to compare the results between zero-inflated models and two-part models were provided in eTable 5 , which indicated that the estimated means and adjusted rate ratios obtained by the two methods were generally comparable in sizes and directions. 
Table 1 Participant Characteristics by Randomised Intervention Groups

\begin{tabular}{|c|c|c|c|}
\hline & CAPICHe intervention $(n=35,535)$ & Usual care control $(n=8883)$ & $\overline{p \text { value }}$ \\
\hline Age (years, SD) & $72.7(12.0)$ & $72.7(11.7)$ & $0.897^{\mathbf{a}}$ \\
\hline \multicolumn{3}{|l|}{ Age group in years $(n, \%)$} & \multirow[t]{6}{*}{$0.821^{\mathrm{b}}$} \\
\hline$\leq 60^{\circ}$ & $4930(13.9 \%)$ & $1226(13.8 \%)$ & \\
\hline $61-70$ & $8509(24.0 \%)$ & $2146(24.2 \%)$ & \\
\hline $71-80$ & $12,014(33.8 \%)$ & $3032(34.1 \%)$ & \\
\hline$>80$ & $10,081(28.4 \%)$ & $2479(27.9 \%)$ & \\
\hline Missing & 1 & 0 & \\
\hline \multicolumn{3}{|l|}{$\operatorname{Sex}(n, \%)$} & \multirow[t]{4}{*}{$0.772^{\mathrm{b}}$} \\
\hline Male & $19,174(54.0 \%)$ & $4778(53.8 \%)$ & \\
\hline Female & $16,360(46.0 \%)$ & $4105(46.2 \%)$ & \\
\hline \multirow{2}{*}{\multicolumn{3}{|c|}{$\begin{array}{l}\text { Missing } \\
\text { Historical total } \operatorname{cost}^{\mathrm{c}}(n, \%)\end{array}$}} & \\
\hline & & & \multirow[t]{4}{*}{$0.317^{\mathrm{b}}$} \\
\hline$\leq \$ 5000$ & $20,909(58.8 \%)$ & $5163(58.1 \%)$ & \\
\hline$\overline{\$ 5001-\$ 25,000}$ & $11,402(32.1 \%)$ & $2875(32.4 \%)$ & \\
\hline$>\$ 25,000$ & $3224(9.1 \%)$ & $845(9.5 \%)$ & \\
\hline \multicolumn{3}{|l|}{ Historical number of admissions ${ }^{\mathrm{d}}(n, \%)$} & \multirow[t]{4}{*}{$0.029^{\mathrm{b}}$} \\
\hline $0-1$ & $21,507(60.5 \%)$ & $5315(59.8 \%)$ & \\
\hline $2-3$ & $10,586(29.8 \%)$ & $2624(29.5 \%)$ & \\
\hline $4+$ & $3442(9.7 \%)$ & $944(10.6 \%)$ & \\
\hline \multicolumn{3}{|l|}{ Mortality } & \multirow[t]{4}{*}{$0.941^{b}$} \\
\hline No & $32,147(90.5 \%)$ & $8034(90.4 \%)$ & \\
\hline Yes & $3387(9.5 \%)$ & $849(9.6 \%)$ & \\
\hline Missing & 1 & 0 & \\
\hline \multicolumn{4}{|l|}{ Overnight admissions ${ }^{\mathrm{e}}(n, \%)$} \\
\hline Nil & $24,601(69.2 \%)$ & $6176(69.5 \%)$ & \multirow{2}{*}{$0.589^{\mathrm{b}}$} \\
\hline At least one admission $(n, \%)$ & $10,934(30.8 \%)$ & $2707(30.5 \%)$ & \\
\hline Mean (SD) admission rate ${ }^{f}$ & $1.810(1.329)$ & $1.825(1.362)$ & \multirow{7}{*}{$\begin{array}{l}0.591^{\mathrm{a}} \\
0.549^{\mathrm{b}}\end{array}$} \\
\hline \multicolumn{3}{|l|}{ Number of overnight admissions ${ }^{\mathrm{f}}$} & \\
\hline Due to medical treatment & $10,157(58.5 \%)$ & $2567(58.9 \%)$ & \\
\hline Due to surgical treatment & $6443(37.1 \%)$ & $1617(37.1 \%)$ & \\
\hline Due to other treatment & $753(4.3 \%)$ & $173(4.0 \%)$ & \\
\hline Missing & 2436 & 584 & \\
\hline Total & 19,789 & 4941 & \\
\hline \multicolumn{4}{|l|}{ Same-day admissions $\mathrm{e}(n, \%)$} \\
\hline Nil & $29,195(82.2 \%)$ & $7233(81.4 \%)$ & \multirow[t]{2}{*}{$0.108^{\mathrm{b}}$} \\
\hline At least one admission $(n, \%)$ & $6340(17.8 \%)$ & $1650(18.6 \%)$ & \\
\hline Mean (SD) admission rate ${ }^{f}$ & $2.893(6.324)$ & $3.467(9.853)$ & $0.025^{\mathrm{a}}$ \\
\hline \multicolumn{3}{|l|}{ Number of same-day admissions ${ }^{\mathrm{f}}$} & \multirow[t]{6}{*}{$<0.001^{\mathrm{b}}$} \\
\hline Due to medical treatment & $4854(50.3 \%)$ & $1599(55.7 \%)$ & \\
\hline Due to surgical treatment & $4224(43.8 \%)$ & $1141(39.7 \%)$ & \\
\hline Due to other treatment & $575(5.9 \%)$ & $131(4.6 \%)$ & \\
\hline Missing ${ }^{\mathrm{g}}$ & 8687 & 2850 & \\
\hline Total & 18,340 & 5721 & \\
\hline
\end{tabular}

Data are mean (SD), $n(\%)$, unless otherwise indicated. ${ }^{a}$ Test difference in means between groups using $t$ tests. ${ }^{b}$ Test difference in proportions between groups using chi-square tests. ${ }^{c}$ Total non-maternity cost for the prior 12 months to randomisation. ${ }^{d}$ Total number of admissions (any type) for the prior 12 months to randomisation. ${ }^{e}$ Admission data for 12 months from randomisation. ${ }^{f}$ Data are for participants who have at least one admission. ${ }^{g}$ Outpatient admissions do not involve a formal admission process to define the type of treatment (medical/surgical/other)

Table 3 presents the costs and rates of same-day admissions with a formal admission process and outpatient admissions (without a formal admission process). The proportions of costs due to same-day admissions with a formal admission process were $92.4 \%$ and $91.4 \%$ for the intervention and control groups, respectively. The proportions of same-day admissions

Table 2 Differences in Health Costs and Service Uses in CAPICHe Intervention Group Relative to Usual Care Control Group

\begin{tabular}{|c|c|c|c|}
\hline \multirow[t]{2}{*}{ Outcome $^{a}$} & \multicolumn{2}{|l|}{ Adjusted results } & \multirow[t]{2}{*}{ Unadjusted results } \\
\hline & Zero-inflated model & Two-part model & \\
\hline $\begin{array}{l}\text { Total non-maternity cost per year }{ }^{1-10} \\
\text { Total overnight admission non-maternity cost per year }{ }^{1-10} \\
\text { Total same-day admission non-maternity cost } \text { per }^{1-10} \text { year }^{1-10} \\
\text { Rate of all admissions per } 1000 \text { person-years }, 1-9 \\
\text { Rate of overnight admissions per } 1000 \text { person-years }{ }^{1-4,6-9} \\
\text { Rate of same-day admissions per } 1000 \text { person-years, } 1-8 \\
\text { Length of hospital stay (day) per } 1000 \text { person-years }{ }^{1-9}\end{array}$ & $\begin{aligned} & \$ 66(-\$ 136 \text { to } \$ 267) \\
& \$ 11(-\$ 172 \text { to } \$ 195) \\
-\$ 40(-\$ 64 \text { to }-\$ 16) & \\
- & 84(-137 \text { to }-31) \\
& 7(-17 \text { to } 31) \\
- & 85(-129 \text { to }-40) \\
& 51(-200 \text { to } 302)\end{aligned}$ & $\begin{array}{l}\$ 59(-\$ 130 \text { to } \$ 238) \\
\$ 76(-\$ 90 \text { to } \$ 232) \\
-\$ 53(-\$ 83 \text { to }-\$ 24) \\
-95(-131 \text { to }-60) \\
8(-9 \text { to } 23) \\
-98(-133 \text { to }-65) \\
\quad 69(-71 \text { to } 201)\end{array}$ & $\begin{array}{l}-\$ 43(-\$ 368 \text { to } \$ 282) \\
-\$ 12(-\$ 301 \text { to } \$ 277) \\
-\$ 60(-\$ 105 \text { to }-\$ 16) \\
-126(-210 \text { to }-41) \\
1(-25 \text { to } 27) \\
-128(-205 \text { to }-52) \\
\quad 11(-299 \text { to } 322)\end{array}$ \\
\hline
\end{tabular}

The analysis is adjusted for ${ }^{1}$ age group (4 categories), ${ }^{2}$ sex, ${ }^{3}$ state of residence, ${ }^{4}$ clinical hierarchy, ${ }^{5}$ batch, ${ }^{6}$ historical cost group (3 categories), 7 historical number of admissions (3 categories), ${ }^{8}$ number of coexist selected diagnosed chronic conditions ( 3 categories), ${ }^{9}$ number of follow-up days from randomisation, and ${ }^{10}$ proportion of surgical treatment (applies to the analysis of cost data only). CAPICHe, Costs to Australian Private Insurance - Coaching Health trial. ${ }^{a}$ All outcomes were obtained in 1 year post-randomisation. Covariates adjusted in the models are noted for each outcome variable. ${ }^{c}$ Using negative binomial model 

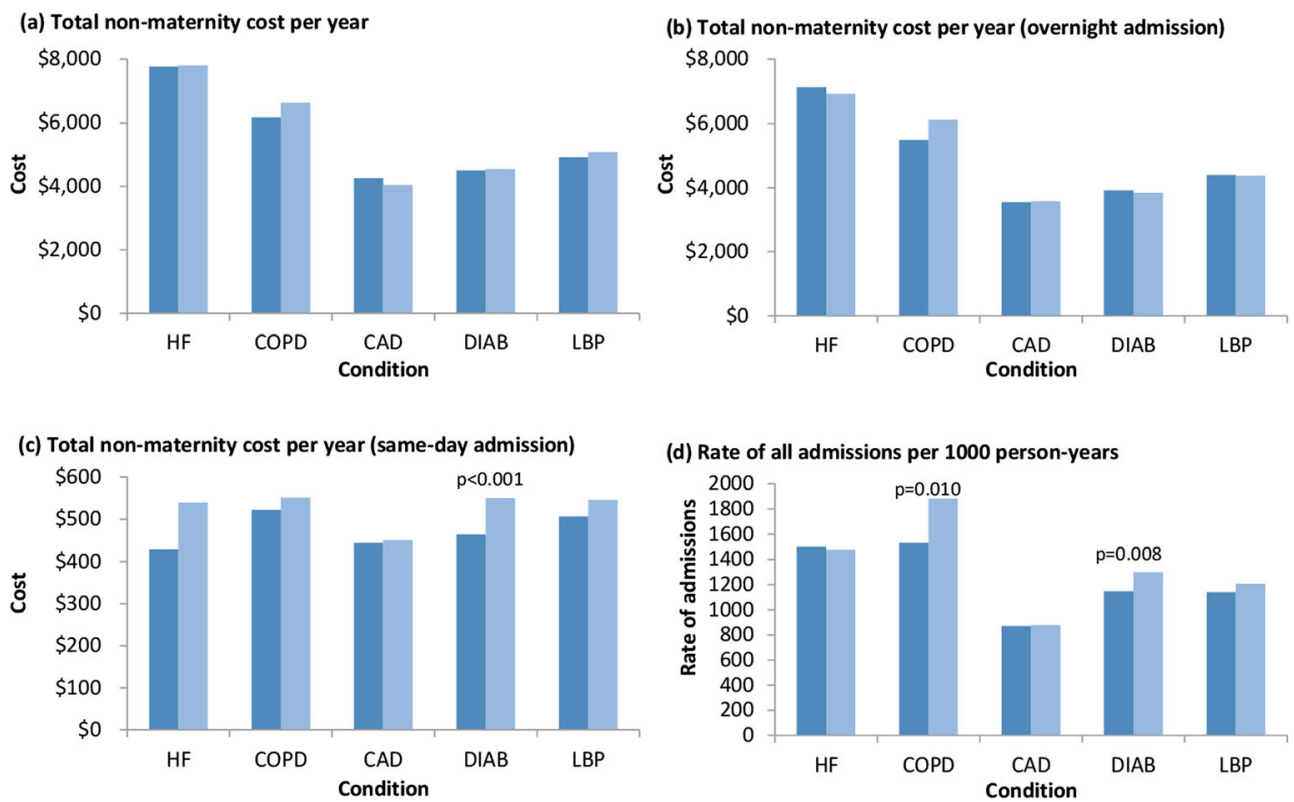

(d) Rate of all admissions per 1000 person-years
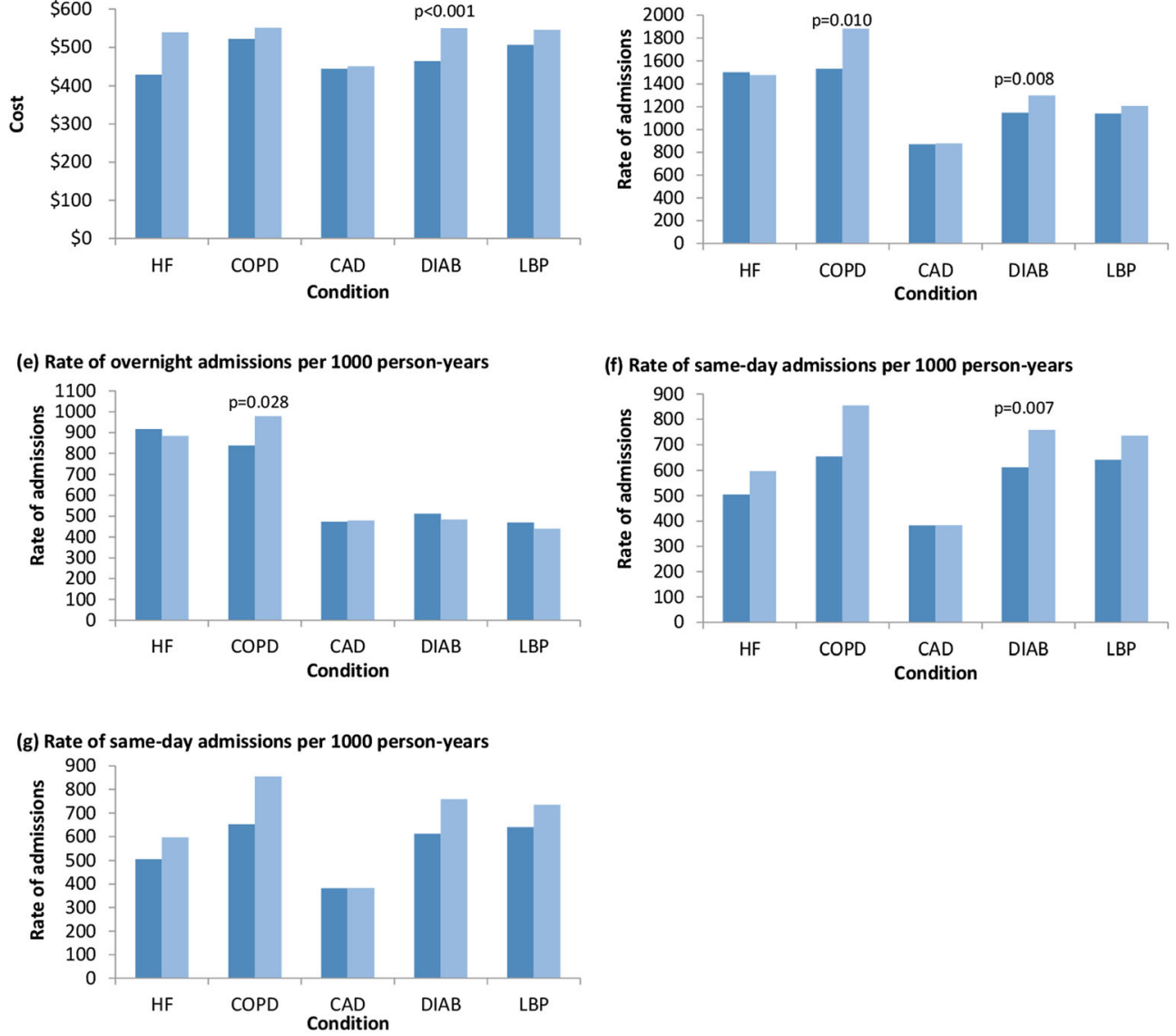

\author{
CAPICHe intervention Usual care control \\ $\mathrm{HF}$, heart failure; COPD, chronic obstructive pulmonary disease; CAD, coronary artery disease; DIAB, diabetes; LBP, low back pain
}

Figure 2. Trial outcomes (subgroup analyses by five chronic conditions).

with a formal admission process were $65.6 \%$ and $65.7 \%$ for the intervention and control groups, respectively. For participants with diabetes, the proportions of same-day admissions with a diabetes procedure were $0.928 \%$ and $0.683 \%$ for the intervention and control groups, respectively.

\section{DISCUSSION}

Based on coaching 15,375 people, this is the largest controlled trial of health coaching to date. In this large intention-to-treat study, the intervention group had total healthcare costs that were $\$ 66$ higher than the control group. This small, $1.3 \%$, difference was not statistically significant. However, this figure does not include the cost of the intervention itself as this is commercial in confidence. Further, there were no significant differences in total cost between the intervention group and the control group for each of the five pre-specified chronic conditions investigated (i.e. heart failure, COPD, coronary artery disease, diabetes, or low back pain). However, the intervention group appeared to have lower cost due to sameday admissions (difference of $\$ 40(7.9 \%) ; p=0.001$ ) and fewer same-day only admissions (difference of 84 per 1000 person-years $(13.8 \%) ; p<0.001)$. Predefined subgroup analyses indicated that for those participants with diabetes in the intervention group, total healthcare costs for same-day admissions were $\$ 86$ lower $(15.6 \% ; p<0.001)$, as a result of fewer 
Table 3 Sample Characteristics for Same-Day Admissions

\begin{tabular}{lll}
\hline \hline Outcome $^{\mathbf{a}}$ & CAPICHe intervention $(\boldsymbol{n = 3 5 , 5 3 5 )}$ & Usual care control $(\boldsymbol{n}=\mathbf{8 8 8 3})$ \\
\hline Total same-day admission non-maternity cost per year & & $\$ 477(23)$ \\
Due to same-day admissions & $\$ 326(9)$ & $\$ 45(7)$ \\
Due to outpatient admissions & $\$ 35(2)$ & $91.4 \%$ \\
Proportion (same-day) & $92.4 \%$ & $426(32)$ \\
Rate of admissions per 1000 person-years & $341(11)$ & $223(35)$ \\
Same-day admissions & $179(10)$ & $65.7 \%$ \\
Outpatient admissions & $65.6 \%$ & $0.683 \%(0.351 \%)$ \\
Proportion (same-day) & $0.928 \%(0.196 \%)$ & \\
Proportion of same-day admissions with a diabetes & & \\
procedure (diabetes participants) & &
\end{tabular}

${ }^{a}$ Data are sample mean (standard error of mean)

same-day only admissions (difference of 146 per 1000 personyears $(19.2 \%) ; p=0.007)$. In addition, the intervention group had fewer admissions (all types) for COPD patients (difference of 351 per 1000 person-years $(18.6 \%) ; p=0.010)$. Upon further analysis, the reduction in same-day admissions could not be attributed to any particular type of admission. It is noted that adjustment can be made to protect against false discovery given the multiple hypothesis tests in subgroup analyses, for example, by controlling for the false discovery rate. ${ }^{14,} 15$ However, in view of the significance levels apart from occurrence by chance, the results would still be robust even with adjustment for multiple hypothesis tests.

This trial had a number of important strengths. It employed a prospective randomised design directly comparing the intervention and usual care and the outcome measures were prespecified and assessed on an intention-to-treat basis. Further, the trial was large with a total of 44,418 individuals ultimately randomised and 15,375 people received health coaching. The most notable weaknesses of the study were the number of participants engaged $(43.3 \%)$ in the intervention group and the number of contacts made per participant over a 12-month period (mean 3.2, median 2, inter-quarter range 2-4). This is relatively low; a systematic review reported interventions lasting 6 to 12 months and those including 12 or more calls produced the most favourable outcomes. ${ }^{16}$ For example, telephone coaching studies in diabetes with at least 14 sessions have shown substantial reductions in glycated haemoglobin (HbAlc). ${ }^{17,} 18$ In addition, no clinical, surrogate, or health outcomes were measured. Changes in $\mathrm{HbAlc}$, blood pressure, cardiac function, pain, maximal oxygen consumption $\left(\mathrm{VO}_{2}\right.$ max), 6-min walk test and frailty; or surrogate outcomes such as medication adherence, patient activity and perceived social support; or health outcomes such as health-related quality of life and capabilities may have demonstrated substantial differences between groups. However, a minimally invasive trial design was established relying on administrative claims data collected on a large sample to address the primary outcome.

There have been several relatively small trials of telephone coaching which have found statistically significant positive effects for participants with diabetes ${ }^{17-19}$, cardiac conditions ${ }^{20}$ and diastolic blood pressure ${ }^{21}$. However, similar numbers of telephone-based coaching trials have reported mixed results on some secondary measures such as improved quality of life but with increased costs and health service use, ${ }^{22,23}$ and other studies have found no discernible intervention effects. ${ }^{24,} 25$ Moreover, these studies have included various designs including different health professionals delivering the intervention, planned versus reactive coaching and supplementary interventions such as occasional face-to-face contact. No single approach has consistently shown a greater benefit than other approaches.

The large study by Wennberg et al. $(N=174,120)$ on which the coaching methods and predictive model used to identify patients in this study were based also reported a relatively small and statistically significant intervention effect of a $3.6 \%$ reduction in medical and pharmacy costs and a $10.1 \%$ reduction in admissions to hospital over a 12-month period, as well as slightly fewer emergency room visits. ${ }^{6}$ That study was a population-based study with participants recruited from seven employers in the USA with health insurance coverage for their employees. Although the Wennberg trial had an initial population of 86,877 in the intervention group, they targeted 22,414 for health coaching of which 9035 (40.3\%) received coaching, and matched these to 3228 of 6805 targeted subjects in the usual care group. ${ }^{6}$ The number of contacts per person (2.24 versus 2.14 ) was similar in both arms. The present trial engaged $43.3 \%$ of the intervention group in coaching. The number of contacts per person is 3.2 and 4.1 for the intervention and the usual care groups, respectively.

Compared with the Wennberg trial, this study had a larger sample size of those who received coaching in the intervention group (9035 vs 15,375) and an older population (37 vs 73 years), and the scope of the outcome measure of costs was more restricted (ancillary claims were not included). ${ }^{6}$ In addition, the CAPICHe trial included low back pain as opposed to asthma in the Wennberg study. Based on the differences in sample size and population age, we expected an intervention effect that was of larger magnitude and greater in statistical significance than reported by Wennberg; ${ }^{6}$ this was not the case.

In conclusion, this large intention-to-treat study did not identify a significant effect on healthcare costs from disease management services through telephone coaching in an older high-risk insured population within 12 months post- 
randomisation. There were no significant differences in total cost between the intervention group and the control group for each of the five pre-specified chronic conditions investigated (i.e. heart failure, COPD, coronary artery disease, diabetes, or low back pain). However, there was some evidence to suggest that the rate of same-day only admissions was significantly lower in the intervention group, especially for participants with diabetes.

Acknowledgements: All authors gratefully acknowledge the contribution of Raimundo Gomes MSc, Bupa Australia, for extracting the data from the Bupa Australia claims database.

Corresponding Author: Paul A. Scuffham, PhD; Centre for Applied Health Economics, Menzies Health Institute Queensland, Griffith University, Brisbane, Australia (e-mail: p.scuffham@griffith.edu.au).

Funding Information This trial was funded by the Bupa Health Foundation, Australia, which is an independent foundation with charity status under the Australian Taxation Office rules.

\section{Compliance with Ethical Standards:}

This study was conducted in accordance with the ethics approval from the Griffith University Human Research Ethics Committe (ref MED/10/ $11 /$ HREC).

Conflict of Interest: PS, JB and SN were independent consultants and have no conflicts of interest. CP and SG are employees of Bupa Australia. DC is an employee of Bupa Health Dialog, part of Bupa Australia. Coaching was provided by Bupa Health Dialog. Health Dialog in the USA provided the risk scoring algorithm which was adapted for Australia.

Disclaimer: The funders had no role in the trial design, data analysis, interpretation of data, or writing of this report. The corresponding author had full access to the extracted data in the trial and had final responsibility for the decision to submit for publication.

\section{REFERENCES}

1. Mays GP, Au M, Claxton G. MARKETWATCH: Convergence And Dissonance: Evolution In Private-Sector Approaches To Disease Management And Care Coordination. Health Aff 2007;26(6): 1683-91.

2. Todd W, Nash D, editors. Disease Management: A Systems Approach to Improving Patient Outcomes. San Francisco: Jossey Bass, 2001.

3. Ellrodt G, Cook DJ, Lee J, et al. Evidence-Based Disease Management. JAMA 1997;278(20):1687-92. https://doi.org/10.1001/jama.1997. 03550200063033

4. Bodenheimer T. Disease management in the American market. BMJ 2000;320(7234):563-6. [published Online First: 2000/02/25]

5. Buntin M, Jain A, Mattke S, et al. Who Gets Disease Management? J Gen Intern Med 2009;24(5):649-55. https://doi.org/10.1007/s11606009-0950-8

6. Wennberg DE, Marr A, Lang $\mathbf{L}$, et al. A Randomized Trial of a Telephone Care-Management Strategy. N Engl J Med 2010;363(13):1245-55. https://doi.org/10.1056/NEJMsa0902321

7. Byrnes J, Goldstein S, Venator B, et al. The impact of population-based disease management services for selected chronic conditions: the Costs to Australian Private Insurance - Coaching Health (CAPICHe) study protocol. BMC Public Health 2012;12(1):114.

8. AIHW. Admitted patient care 2014-15: Australian hospital statistics. Health services series no. 68. Cat. no. HSE 172. Canberra: AIHW. 2016

9. Little RJ, Long $\mathbf{Q}$, Lin $\mathbf{X}$. A comparison of methods for estimating the causal effect of a treatment in randomized clinical trials subject to noncompliance. Biometrics 2009;65:640-9. https://doi.org/10.1111/j. 1541-0420.2008.01066.x.

10. Sussman JB, Hayward RA. An IV for the RCT: using instrumental variables to adjust for treatment contamination in randomised controlled trials. BMJ 2010;340:c2073. https://doi.org/10.1136/bmj.c2073

11. Akbarzadeh Baghban A, Pourhoseingholi A, Zayeri F, et al. Zero inflated statistical count models for analysing the costs imposed by GERD and dyspepsia. Arab J Gastroenterol 2013;14(4):165-68. doi:https://doi.org/10.1016/j.ajg.2013.09.004

12. Greene $\mathbf{W}$. Accounting for excess zeros and sample selection in poisson and negative binomial regression models. Working Papers EC-94-10. New York University: New York University, Leonard N. Stern School of Business, Department of Economics, 1994.

13. Mihaylova B, Briggs A, O'Hagan A, et al. Review of statistical methods for analysing healthcare resources and costs. Health Econ 2011;20(8):897-916. https://doi.org/10.1002/hec.1653 [published Online First: 2010/08/28]

14. Ng SK, Holden L, Sun J. Identifying comorbidity patterns of health conditions via cluster analysis of pairwise concordance statistics. Stat Med 2012;31:3393-405. https://doi.org/10.1002/sim.5426

15. Ng SK. A two-way clustering framework to identify disparities in multimorbidity patterns of mental and physical health conditions among Australians. Stat Med 2015;34:3444-60. https://doi.org/10.1002/sim. 6542

16. Eakin EG, Lawler SP, Vandelanotte C, et al. Telephone interventions for physical activity and dietary behavior change: a systematic review. Am J Prev Med 2007;32(5):419-34. https://doi.org/10.1016/j.amepre.2007. 01.004 [published Online First: 2007/05/05]

17. Williams ED, Bird D, Forbes AW, et al. Randomised controlled trial of an automated, interactive telephone intervention (TLC Diabetes) to improve type 2 diabetes management: baseline findings and six-month outcomes. BMC Public Health 2012;12:602. https://doi.org/10.1186/1471-245812-602 [published Online First: 2012/08/04]

18. Wolever RQ, Dreusicke M, Fikkan J, et al. Integrative health coaching for patients with type 2 diabetes: a randomized clinical trial. Diabetes Educ 2010;36(4):629-39. https://doi.org/10.1177/0145721710371523 [published Online First: 2010/06/11]

19. Gordon LG, Bird D, Oldenburg B, et al. A cost-effectiveness analysis of a telephone-linked care intervention for individuals with Type 2 diabetes. Diabetes Res Clin Pract 2014;104(1):103-11. https://doi.org/10.1016/j. diabres.2013.12.032 [published Online First: 2014/02/08]

20. Sangster $\mathbf{J}$, Church $\mathbf{J}$, Haas $\mathbf{M}$, et al. A Comparison of the Costeffectiveness of Two Pedometer-based Telephone Coaching Programs for People with Cardiac Disease. Heart Lung Circ 2015;24(5):471-9. https://doi.org/10.1016/j.hlc.2015.01.008 [published Online First: 2015/02/24]

21. Patja K, Absetz P, Auvinen A, et al. Health coaching by telephony to support self-care in chronic diseases: clinical outcomes from The TERVA randomized controlled trial. BMC Health Serv Res 2012;12:147. https://doi.org/10.1186/1472-6963-12-147 [published Online First: 2012/06/12]

22. Hawkes AL, Patrao TA, Atherton J, et al. Effect of a telephone-delivered coronary heart disease secondary prevention program (proactive heart) on quality of life and health behaviours: primary outcomes of a randomised controlled trial. Int $\mathrm{J}$ Behav Med 2013;20(3):413-24. https://doi.org/10.1007/s12529-012-9250-5 [published Online First: 2012/09/27]

23. Turkstra E, Hawkes AL, Oldenburg B, et al. Cost-effectiveness of a coronary heart disease secondary prevention program in patients with myocardial infarction: results from a randomised controlled trial (ProActive Heart). BMC Cardiovasc Disord 2013;13:33. https://doi.org/10. 1186/1471-2261-13-33 [published Online First: 2013/05/03]

24. Blackberry ID, Furler JS, Best JD, et al. Effectiveness of general practice based, practice nurse led telephone coaching on glycaemic control of type 2 diabetes: the Patient Engagement and Coaching for Health $(\mathrm{PEACH})$ pragmatic cluster randomised controlled trial. BMJ 2013;347:f5272. https://doi.org/10.1136/bmj.f5272 [published Online First: 2013/09/21]

25. Ruggiero L, Riley BB, Hernandez R, et al. Medical assistant coaching to support diabetes self-care among low-income racial/ethnic minority populations: randomized controlled trial. West J Nurs Res 2014;36(9):1052-73. https://doi.org/10.1177/0193945914522862 [published Online First: 2014/02/27] 